\title{
Prospective phase II trial of regional hyperthermia and whole liver irradiation for numerous chemorefractory liver metastases from colorectal cancer
}

Jeong II Yu, MD¹, Hee Chul Park, MD, PhD ${ }^{1,2}$, Doo Ho Choi, MD, PhD1, Jae Myoung Noh, MD', Dongryul Oh, MD', Jun Su Park, MD', Ji Hyun Chang, MD³, Seung Tae Kim, MD', Jeeyun Lee, MD, PhD ${ }^{4}$, Se Hoon Park, MD', Joon Oh Park, MD, Young Suk Park, MD, Ho Yeong Lim, MD, PhD , Won Ki Kang, MD ${ }^{4}$

\begin{abstract}
${ }^{1}$ Department of Radiation Oncology, Samsung Medical Center, Sungkyunkwan University School of Medicine, Seoul;
${ }^{2}$ Department of Medical Device Management and Research, Samsung Advanced Institute for Health Sciences and Technology, Sungkyunkwan University, Seoul;

${ }^{3}$ Department of Radiation Oncology, Seoul St. Mary's Hospital, The Catholic University of Korea College of Medicine, Seoul;

${ }^{4}$ Department of Medicine, Samsung Medical Center, Sungkyunkwan University School of Medicine, Seoul, Korea
\end{abstract}

Purpose: A prospective phase II trial was conducted to evaluate the effectiveness and toxicity of regional hyperthermia and whole liver irradiation (WLI) for numerous chemorefractory liver metastases from colorectal cancer.

Materials and Methods: Enrolled patients had numerous chemorefractory hepatic metastases from colorectal cancer. Five sessions of hyperthermia and seven fractions of 3-gray WLI were planned. Health-related quality of life (HROLL) was determined using the Korean version of the European Organization for Research and Treatment of Cancer quality of life questionnaire C-30 and the Functional Assessment of Cancer Therapy-Hepatobiliary version 4.0. Objective and pain response was evaluated.

Results: A total of 12 patients consented to the study and the 10 who received WLI and hyperthermia were analyzed. WLI was completed as planned in nine patients and hyperthermia in eight. Pain response was partial in four patients and stable in four. Partial objective response was achieved in three patients (30.0\%) and stable disease was seen in four patients at the 1-month follow-up. One patient died 1 month after treatment because of respiratory failure related to pleural metastasis progression. Other grade III or higher toxicities were detected in three patients; however, all severe toxicities were related to disease progression rather than treatment. No significant difference in $\mathrm{HROOL}$ was noted at the time of assessment for patients who were available for questionnaires.

Conclusion: Combined WLI and hyperthermia were well tolerated without severe treatment-related toxicity with a promising response from numerous chemorefractory hepatic metastases from colorectal cancer.

Keywords: Colorectal neoplasms, Radiotherapy, Induced hyperthermia, Quality of life, Pain

Received 18 December 2015, Revised 5 February 2016, Accepted 3 March 2016.

Correspondence: Hee Chul Park, MD, PhD, Department of Radiation Oncology, Samsung Medical Center, Sungkyunkwan University School of Medicine, 81 Irwon-ro, Gangnam-gu, Seoul 06351, Korea. Tel: +82-2-3410-2612, Fax: +82-2-3410-2619, E-mail: hee.ro.park@samsung.com

(c) This is an Open Access article distributed under the terms of the Creative Commons Attribution Non-Commercial License (http://creativecommons.org/ licenses/by-nc/4.0/) which permits unrestricted non-commercial use, distribution, and reproduction in any medium, provided the original work is properly cited.

www.e-roj.org 


\section{Introduction}

The liver is the most common metastatic site of gastrointestinal tract malignancies, which are associated with the blood supply from the portal circulation [1]. About 25\% of patients with colorectal cancer are initially diagnosed with hepatic metastasis and metachronous liver metastasis is detected during followup in another 50\% initially diagnosed with localized disease [2]. A selected population of about $20 \%$ to $25 \%$ of patients have indications for surgical resection; however, about two-thirds of curatively resected patients show liver recurrence within 2 years $[3,4]$.

Systemic or hepatic arterial infusion chemotherapy is the mainstay treatment for hepatic metastasis, even for curatively resected patients [4-6]. With the development and appropriate selection of chemotherapy, survival outcomes are improving in these patients [7]. However, under current standard intensive chemotherapy, intrahepatic progression proceeds within 10 months $[5,6]$. Though an additional regimen of chemotherapy can be considered in selected patients [8], it is not indicated for a subset of patients because of liver function, performance status, or other factors [9].

Whole liver irradiation (WLI) is a palliative option for extensive liver metastases that cause pain from stretching of the liver capsule and deterioration of liver function [10]. Though WLI shows acceptable efficacy for symptom relief in $55 \%$ to $80 \%$ of patients [11], local control is disappointing because of the limitations of deliverable radiation doses associated with the intrinsic radiosensitivity of the liver [12]. To enhance WLI results, concurrent radiosensitizers or chemotherapy have been examined [13]. However, outcomes are only modestly affected with significant increases in toxicity.

Hyperthermia is a well-known adjunct radiation therapy (RT) modality for breast, cervix, and rectal cancer [14]. Theoretically, hyperthermia improves the killing of tumor cells known to be radioresistant. In addition, several randomized controlled trials showed benefits to locoregional control through the promotion of radiosensitizing effects [15].

Based on this background, we designed a phase II trial to evaluate the effectiveness and toxicity of WLI plus hyperthermia for patients with numerous chemorefractory hepatic metastases from colorectal cancer.

\section{Materials and Methods}

The Institutional Review Board approved this study (IRB No.
2013-06-040) and all patients provided written informed consent before enrollment. This trial is registered at clinicaltrials.gov (NCT01963117). The protocol and Consolidated Standards of Reporting Trials (CONSORT) checklist of the present trial are available in the supporting information.

\section{Patients}

All patients were required to have pathologically confirmed colorectal cancer with numerous unresectable and chemorefractory hepatic metastases. Patients were recruited to the present study according to the following eligibility criteria: age 20 years or older; adequate bone marrow function (hemoglobin $>8 \mathrm{~g} / \mathrm{dL}$; absolute neutrophil count $>1,500$ / $\mu \mathrm{L}$; platelet count $>50,000 / \mu \mathrm{L}$ ), adequate liver function (prothrombin time-international normalized ratio $<1.7$; albumin $\geq 2.8 \mathrm{~g} / \mathrm{dL}$, aspartate or alanine aminotransferase $<6$ times). We excluded patients who had fewer than 8 weeks of expected survival, received previous upper abdominal RT, or had uncontrolled ascites, unstable respiration related to pleural effusion, or chronic obstructive pulmonary disease. Patients with grade 4 Eastern Cooperative Oncology Group (ECOG) performance status were also excluded. All possible indications of the study were discussed before registration by a colorectal tumor board of surgeons, gastroenterologists, medical oncologists, and radiation oncologists.

The protocol was amended once on January 2014 due to slow accrual of patients, which extended the indication from primary colorectal cancer to primary gastro-intestinal malignancy, but only colorectal malignancies were ultimately enrolled.

\section{Radiation therapy}

To avoid missing targets related to respiratory motion, fullimage guidance protocols were used in all patients. Respiration training was conducted with audiovisual coaching before simulation to improve respiration periodicity and audiovisual respiration coaching was used in simulations and $R T$ treatment delivery. Simulations used GE LightSpeed plus 16 scanners (GE Healthcare, Milwaukee, WI, USA). Intravenous contrast media (Visipaque 270; Amersham Health Centre, Amersham, UK; $2 \mathrm{~mL} / \mathrm{kg}$ for maximum $200 \mathrm{~mL}$ ) was injected at $5 \mathrm{~mL} / \mathrm{s}$. Exhale breath-hold computed tomography (CT) scans were acquired at 50 to 60 seconds afterward. Subsequently, fourdimensional CT scans were repeated using the Real-time Position Management system (Varian Medical Systems, Palo Alto, CA, USA).

Clinical target volume (CTV) was defined as the entire liver 
on CT images. Internal target volume (ITV) was defined as the sum of CTVs for all respiration phases. Planning target volume was defined as ITV plus a $5-\mathrm{mm}$ setup margin. Maximum conformal planning was made using 3-5 coplanar or noncoplanar beams of 4 or 10 MV X-ray with field-in-field technique.

Radiation doses were fixed at 21 gray (Gy) in 7 fractions using the protocol of the previous Radiation Therapy Oncology Group (RTOG) trial [16].

\section{Hyperthermia}

Hyperthermia therapy was delivered via a Celsius TCS electrohyperthermia system (Celsius42+ GmbH, Cologne, Germany) for 60 minutes twice a week, at intervals of at least 72 hours, for 5 total sessions from date of simulation. In the third session, hyperthermia was conducted immediately after the first WLI fraction; the fourth and fifth sessions were also delivered after WLI using a twice-a-week protocol. As much as possible, the entire liver was encompassed within a $20 \times$ $20 \mathrm{~cm}^{2}$ aperture hyperthermia region. The energy escalation scheme is presented in Supplementary Table 1. In this machine, to reduce the possibility of skin burns, a cooling system was provided and activated during the hyperthermia session. Skin temperature was continuously monitored using three glass fiber-optic sensors (Celsius TempSens; Celsius42+ GmbH) during hyperthermia sessions.

\section{Health-related quality of life assessment}

Health-related quality of life (HROoL) was assessed at baseline and 1, 2, and 3 months post treatment using the Korean version of European Organization for Research and Treatment of Cancer quality of life questionnaire C-30 (EORTC QLO-C30) and Functional Assessment of Cancer Therapy-Hepatobiliary version 4.0 (FACT-Hep v4.0). At the time of HROoL assessment, current pain status was also evaluated with a visual analog scale (VAS) for pain.

\section{Follow-up and toxicity evaluation}

The first follow-up evaluation was planned for 1 month after WLI plus hyperthermia treatment, then every month for 3 months. At each follow-up evaluation, patients underwent clinical, and physical examinations. HRQoL, VAS for pain, and carcinoembryonic antigen (CEA) were evaluated. CT scanning was performed, and chemistry profiling including liver function was checked.

Toxicity levels were assessed with the Common Terminology Criteria for Adverse Events version 4.0.

\section{Statistical analysis}

This trial was planned as a phase II study. We planned to enroll 47 patients in order to observe a 50\% symptom palliation rate which was defined as complete or partial pain response on 1 month follow-up after WLI plus hyperthermia. The study was estimated to have $80 \%$ power to detect a $20 \%$ difference from WLI alone at a 5\% two-sided significance level.

Pain response, as a primary endpoint, was evaluated at the 1 month follow-up with International Bone Metastases Consensus Group (IBMCG) criteria (Supplementary Table 2). Objective responses were evaluated at the 1 month followup with the revised Response Evaluation Criteria in Solid Tumors (RECIST v1.1). Repeated measures one-way analysis of variance (ANOVA) was used to determine changes in HROoL. Distributions of time-to-event variables were estimated using the Kaplan-Meier product-limit method and compared using the log-rank test. Local progression-free survival (LPFS), which is defined as progression within liver according to RECIST v1.1 and pain progression-free survival (PPFS), which is defined as progression according to IBMCG criteria, was calculated from date of WLI start to date of event developed or the last followup visit.

\section{Results}

\section{Patients}

The study ended early because accrual was lower than expected: 12 patients consented and were enrolled from November 2013 to August 2014. One refused further treatment before simulation and another did not receive irradiation after simulation because of progression of brain metastasis. Followup was available for 10 patients at 1 month, five at 2 months, and four patients at 3 months after WLI plus hyperthermia (Fig 1), and the 10 patients who received WLI were analyzed.

Demographic and baseline characteristics of the 10 enrolled patients who received treatment are presented in Table 1 . The median age was 55 years (range, 44 to 80 years). One patient had an ECOG performance status 2. Neuroendocrine carcinoma was found in 20\%. All enrolled patients had a history of progression after at least one regimen of chemotherapy.

\section{Previous treatment}

The median number of administered chemotherapy cycles before study registration was 23 (range, 1 to 46). More than 10 cycles of chemotherapy were used except in two patients with extensive hepatic metastasis progression after the start of the first chemotherapy regimen. These patients could not receive 


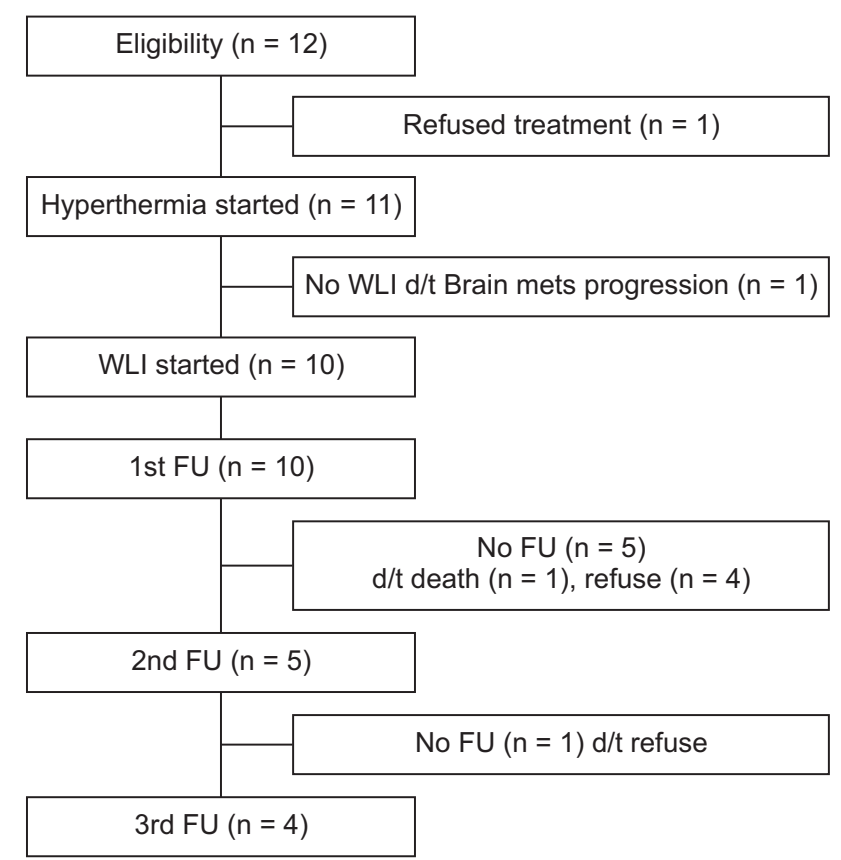

Fig. 1. Consolidated Standards of Reporting Trials (CONSORT) diagram. WLI, whole liver irradiation; FU, follow-up.

further chemotherapy because of liver function deterioration. The commonly used chemotherapy regimens were capecitabine and oxaliplatin or 5-flurouracil, with leucovorin and irinotecan for adenocarcinoma or etoposide with cisplatin or octreotide for neuroendocrine carcinoma. Detailed chemotherapy regimens are displayed in Supplementary Table 3. Palliative RT was given to two patients with primary masses and one with spine metastasis with spinal cord compression.

\section{Whole hepatic irradiation plus hyperthermia}

Except for the patient who refused further treatment after 6 fractions of WLI, all patients completed the planned total dose of $21 \mathrm{~Gy}$. The planned 5 hyperthermia sessions were completed in eight patients, although a 10\% energy reduction was needed in two because of hyperthermia-related pain. Another two patients refused further hyperthermia after the third and fourth sessions because of pain. During hyperthermia, skin surface temperatures were maintained at $36^{\circ} \mathrm{C}$ to $37.5^{\circ} \mathrm{C}$ in all patients.

\section{Pain response}

Of the 10 patients who received WLI plus hyperthermia, at 1 month, a partial response (PR) was seen in four patients with stable disease. At 2 months, one patient with PR converted to complete response, two patients showed progressive disease
Table 1. Patient characteristics

\begin{tabular}{lc}
\hline \multicolumn{1}{c}{ Variable } & $\begin{array}{c}\text { No. of patients } \\
(\%)\end{array}$ \\
\hline Age (yr), median (range) & $55(44-80)$ \\
Sex & \\
Male & $5(50.0)$ \\
Female & $5(50.0)$ \\
ECOG performance status & \\
0 & $8(80.0)$ \\
1 & $1(10.0)$ \\
2 & $1(10.0)$ \\
Pathology & \\
Adenocarcinoma & $8(80.0)$ \\
Neuroendocrine carcinoma & $2(20.0)$ \\
Site of primary tumor & \\
$\quad$ Colon, other & $2(20.0)$ \\
Colon, sigmoid & $3(30.0)$ \\
Rectum & $5(50.0)$ \\
Time to hepatic metastasis & \\
Synchronous & $7(70.0)$ \\
Metachronous & $3(30.0)$ \\
Primary tumor resection & \\
Initial & $4(40.0)$ \\
After chemotherapy & $1(10.0)$ \\
Not done & $5(40.0)$ \\
Previous chemotherapy lines (repeated measures) & \\
First & $10(100.0)$ \\
Second & $9(90.0)$ \\
Third & $8(80.0)$ \\
Fourth or more & $6(60.0)$ \\
\hline ECOG, Easter Coopra &
\end{tabular}

ECOG, Eastern Cooperative Oncology Group.

(PD), and one patient showed stable disease. No change in pain was noticed at 3 months. The median VAS score was 4.0 (range, 0 to 10), 3.5 (range, 0 to 7), 3.0 (range, 0 to 7), and 0 (range, 0 to 9 ) before and at 1, 2, and 3 months after RT, respectively.

\section{Objective response of hepatic metastasis}

Of the 10 patients who received WLI plus hyperthermia, PD of hepatic metastasis was noted in two patients (20\%), and three showed PR (Fig. 2). At 2 months, the other three showed hepatic PD, and three remained PD-free 3 months after treatment.

\section{Laboratory change and adverse events after WLI and hypothermia}

During the treatment period and follow-up, grade III or higher toxicities were detected in four patients. Laboratory change from baseline to 1, 2 and 3 months after hyperthermia and 

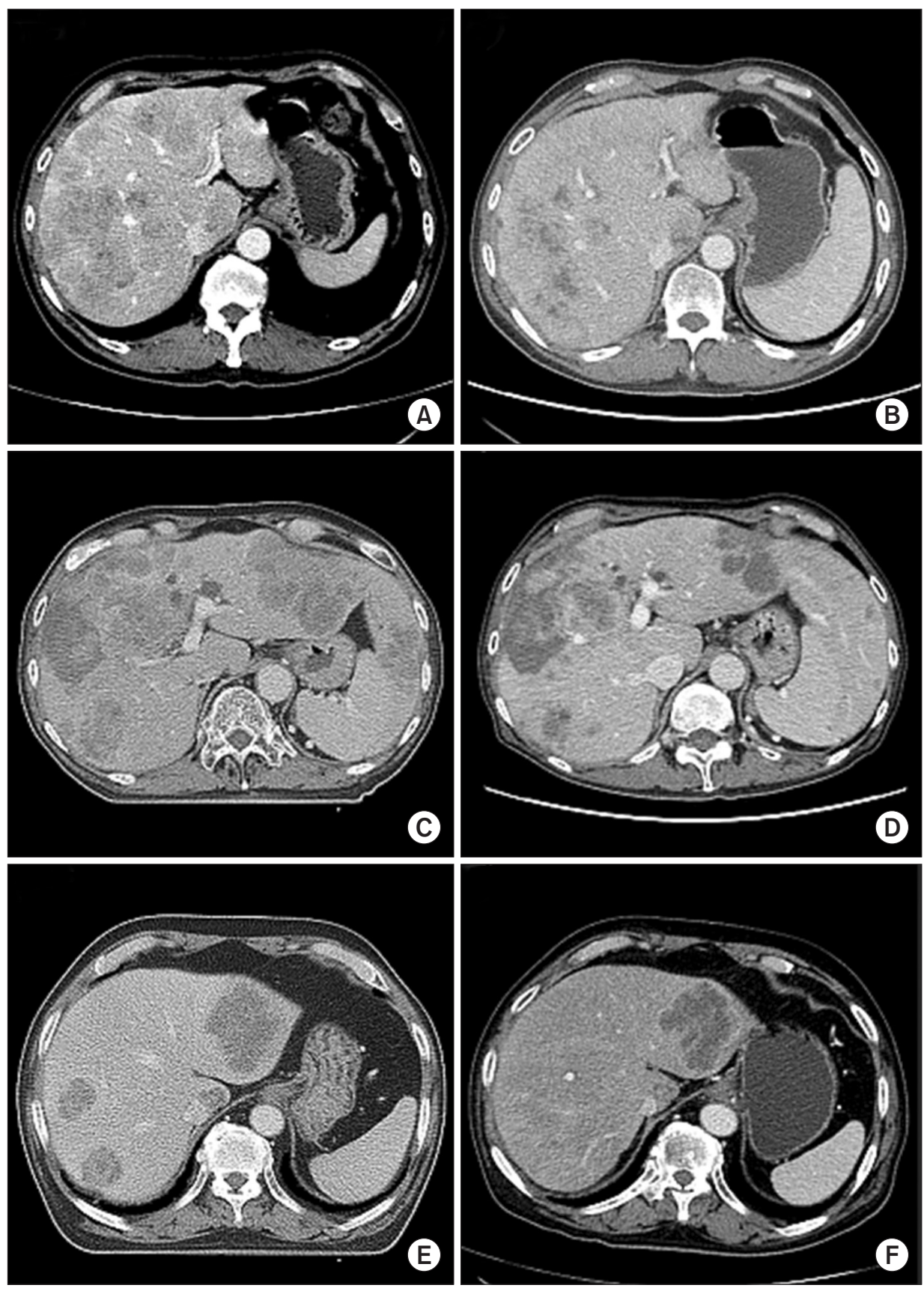

Fig. 2. Changes in computed tomography (CT) images after whole liver irradiation (WLI) plus hyperthermia: three of 10 patients showed partial response 1 month after treatment. CT images taken before $(A, C, E)$ and 1 month after WLI $(B, D, F)$.

WLI is displayed in Table 2

\section{HROoL}

HRQoL assessment was possible in only a small portion of enrolled patients due to loss to follow-up. HROoL was assessed at baseline in all patients, at 1 month in five, at 2 months in four and at 3 months in four.

No significant differences in HRQoL were noted at the time of assessment in patients who could answer the questionnaire, both EORTC OLQ-C3O (Fig. 3) and FACT-Hep (Fig. 4). HRQoL score generally tended to improve with follow-up. However, global health status and EORTC OLO-C30 symptom scales decreased slightly during follow-up.

\section{Local progression-free survival and pain progression-} free survival

At 3 months, LPFS was 30\% and PPFS was 58.3\%. Possible prognostic factors related to survival rates are in Table 3. Elevation of Child-Pugh score ( $p=0.04)$ (Fig. 5A) at 1 month after WLI plus hyperthermia was a significant prognostic 
Table 2. Laboratory change after WLI and hyperthermia

\begin{tabular}{|c|c|c|c|c|c|c|c|}
\hline & \multirow{2}{*}{ Baseline } & \multicolumn{6}{|c|}{ Follow-up after treatment } \\
\hline & & $1 \mathrm{mo}$ & $p$-value & $2 \mathrm{mo}$ & $p$-value & $3 \mathrm{mo}$ & $p$-value \\
\hline Hemoglobin & $10.8(8.7-13.9)$ & $10.9(7.7-14.2)$ & 0.15 & $10.9(8.7-13.1)$ & 1.0 & $11.6(9.9-10.5)$ & 0.25 \\
\hline Platelet & $232(132-560)$ & $121(40-227)$ & 0.008 & 138 (54-209) & 0.13 & 241 (115-329) & 0.13 \\
\hline AST & $46(16-84)$ & $51(28-146)$ & 0.38 & $54(40-96)$ & 0.44 & $44(39-51)$ & 1.00 \\
\hline ALT & $36(12-64)$ & $25(11-83)$ & 0.72 & $36(11-82)$ & 0.56 & $45(16-60)$ & 1.00 \\
\hline Albumin & $3.9(2.4-4.8)$ & $3.7(2.4-4.7)$ & 0.08 & $3.3(2.4-4.3)$ & 0.31 & $4.2(3.7-4.5)$ & 0.63 \\
\hline Total bilirubin & $0.8(0.5-4.8)$ & $1.1(0.3-13.8)$ & 0.48 & $0.6(0.4-2.6)$ & 0.53 & $0.6(0.4-0.8)$ & 0.25 \\
\hline Creatinine & $0.72(0.59-1.09)$ & $0.65(0.46-0.97)$ & 0.002 & $0.76(0.64-0.98)$ & 0.75 & $0.71(0.57-0.85)$ & 0.13 \\
\hline
\end{tabular}

WLI, whole liver irradiation; AST, aspartate aminotransferase; ALT, alanine amainotransferase.

A

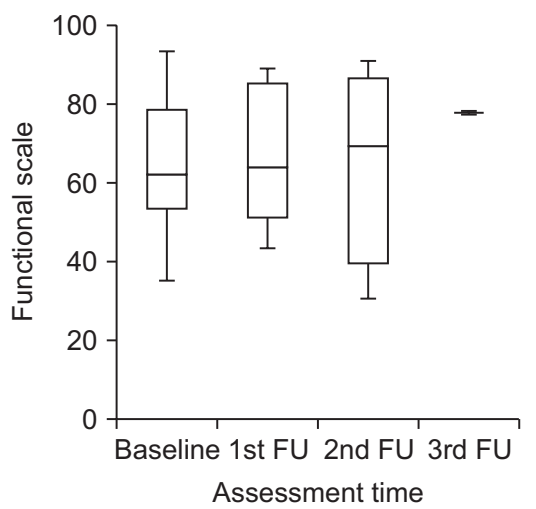

B

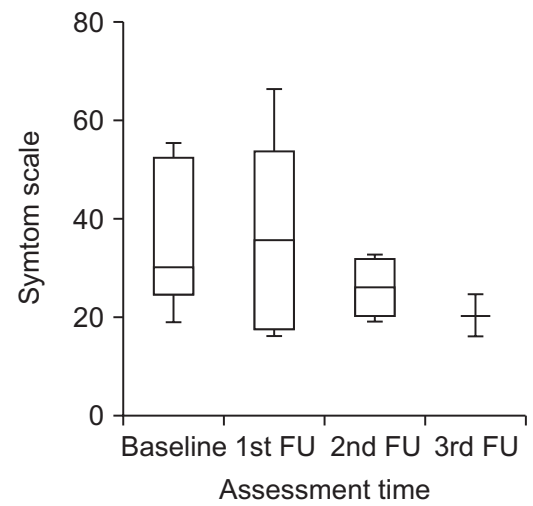

C

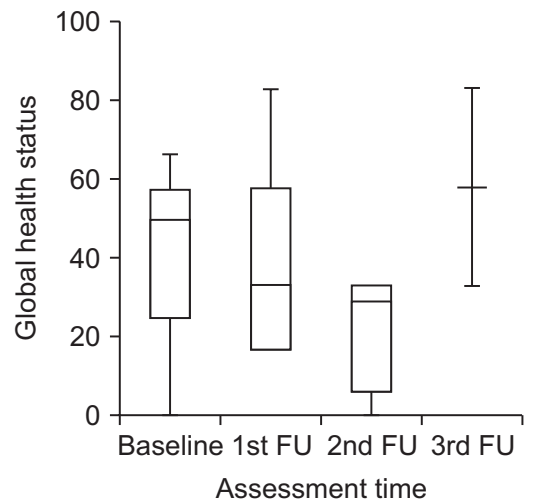

Fig. 3. Change in EORTC QLQ-C30 (European Organization for Research and Treatment of Cancer quality of life questionnaire C-30) subscales from baseline to 3 months after whole liver irradiation plus hyperthermia. (A) Functional scales, (B) symptom scale, and (C) global health status.

factor for LPFS. LPFS tended to be higher in patients without combined extrahepatic metastasis ( $p=0.1$ ) (Supplementary Fig. 1).

Whole liver volume larger than $3,000 \mathrm{~mL}(p=0.02)$ (Fig. $5 B)$, which indirectly represented metastatic tumor volume, was significantly related to lower PPFS. PPFS tended to be higher in patients with neuroendocrine carcinoma $(p=0.11)$ (Supplementary Fig. 2) and initial CEA level higher than $50 \mathrm{ng} /$ $m L(p=0.12)$ (Supplementary Fig. 3). Objective response at 1 month was not related to PPFS ( $p=0.43)$.

\section{Discussion and Conclusion}

The liver is a radiosensitive organ. Radiation-induced hepatic toxicity is still an unresolved problem in liver-directed RT [17]. Liver function deterioration after palliative WLI in patients with uncontrollable chemorefractory hepatic metastasis remains a concern of radiation oncologists.

Recent studies have shown that the incidence is decreasing, but radiation-induced hepatic toxicity develops in a small subset of patients who receive RT for primary liver cancer even after low-dose RT [18]. However, the conditions of normal liver, metastatic liver cancer and primary liver cancer are clearly different, because most primary liver cancers develop from chronic liver disease such as cirrhosis or viral hepatitis [19]. Because liver function deterioration in hepatic metastasis originates from the metastatic tumor burden, liver function is improved after WLI rather than being compromised [12]. A prospective RTOG study showed no radiation-induced hepatic toxicity in 109 patients with liver metastasis after low-dose WLI such as 21 Gy in 7 fractions [16].

Low-dose WLI is also an effective palliative modality for abdominal pain, nausea/vomiting and other conditions [11]. According to the literature, therefore, low-dose WLI should be considered to maintain $\mathrm{HROOL}$ in patients with symptomatic liver metastasis. In fact, the Liver Metastases Consensus Group suggests WLI is safe and effective for symptomatic liver metastases, based on a review of available clinical data [6]. 
A

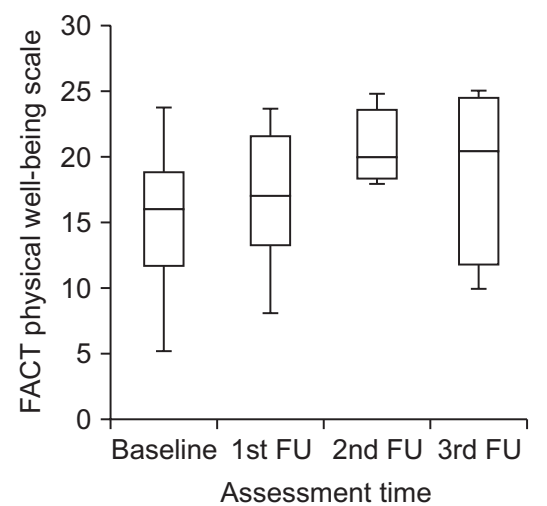

D

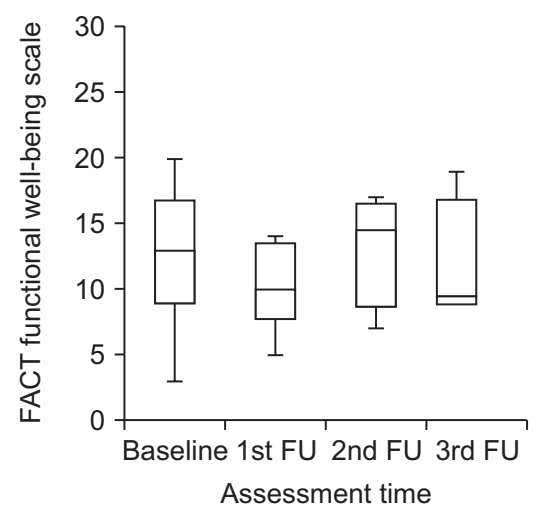

G

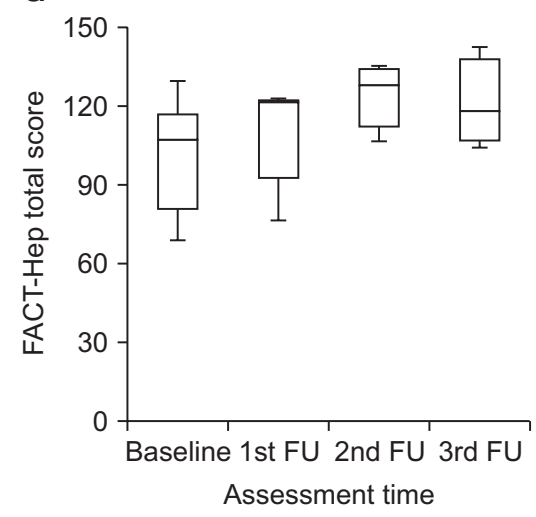

B
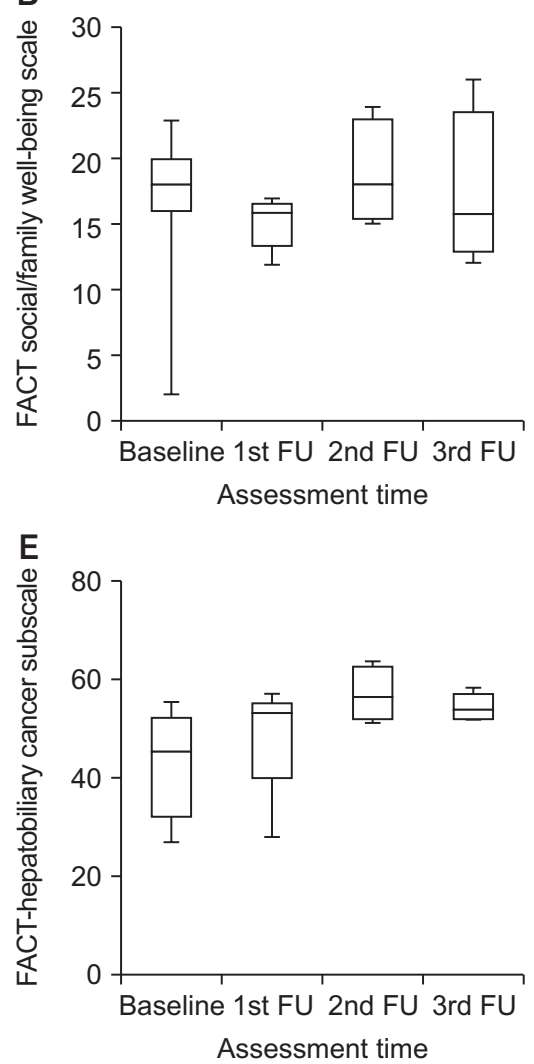

C

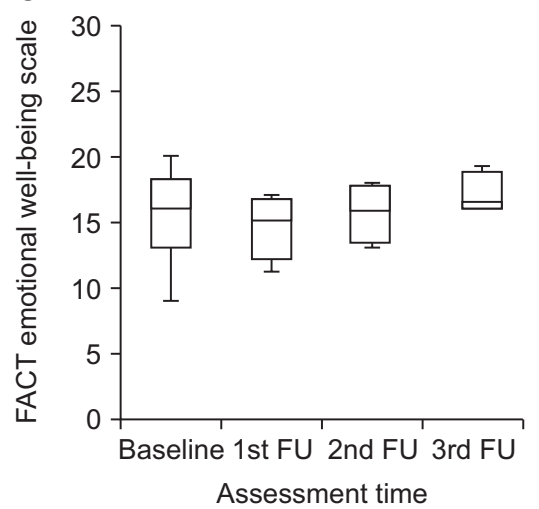

$\mathrm{F}$

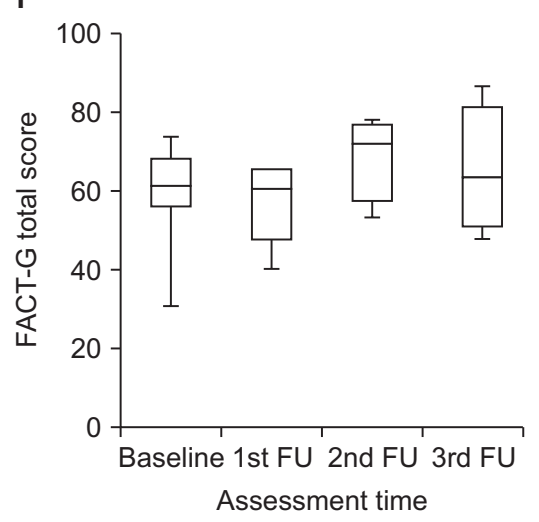

Our group previously reported the results of a retrospective study of WLI as 21 Gy in 7 fractions in 16 patients with hepatic metastasis of colorectal cancer [20]. Palliation of abdominal pain was acceptable, but median overall survival was only 9 weeks. Objective response evaluation was not possible because of short follow-up. According to other studies using the same WLI dose, only a minimal response is achieved in a small portion of patients [12]. Although low-dose WLI is an effective palliative modality, a method that can improve local response and maintain RT effects in the normal liver is needed. Therefore, we conducted a prospective phase II trial
Fig. 4. Change in FACT-Hep (Functional Assessment of Cancer Therapy-Hepatobiliary) subscales from baseline to 3 months after whole liver irradiation plus hyperthermia: (A) physical well-being, (B) social and family well-being, (C) emotional well-being, (D) functional well-being, (E) hepatobiliary cancer subscale, (F) FACTGeneral, and (G) FACT-Hep total scores. FU, follow-up.

to evaluate the response and adverse events from combined WLI and hyperthermia for chemorefractory hepatic metastasis from colorectal cancer.

Among radiation oncology experts, hyperthermia is a wellrecognized radiosensitizer. Hyperthermia is effective in $\mathrm{S}$ phase, low $\mathrm{pO}_{2}$, low $\mathrm{pH}$, and low perfusion areas that are known to be radioresistant [15]. Improvement in locoregional control via combined RT and hyperthermia has been reported at several cancer sites, such as the breast, cervix, and head and neck [14].

Similar to the results of other prospective and retrospective low-dose WLI studies $[12,16,20]$, most patients in our trial 
Table 3. Possible prognostic factors for LPFS and PPFS

\begin{tabular}{|c|c|c|c|c|}
\hline Variable & LPFS at 3 mo (\%) & $p$-value & PPFS at $3 \mathrm{mo}(\%)$ & $p$-value \\
\hline Sex & & 0.15 & & 0.96 \\
\hline Male & 30.0 & & 80.0 & \\
\hline Female & 66.7 & & 37.5 & \\
\hline Age (yr) & & 0.10 & & 0.81 \\
\hline$>55$ & 53.3 & & 75.0 & \\
\hline$\leq 54$ & 0.0 & & 53.3 & \\
\hline ECOG performance status & & 0.24 & & 0.33 \\
\hline 0 & 41.7 & & 47.6 & \\
\hline $1-2$ & 0.0 & & NR & \\
\hline Primary site & & 0.31 & & 0.65 \\
\hline Colon & 0.0 & & 75.0 & \\
\hline Rectum & 50.0 & & 53.3 & \\
\hline Pathology & & 0.28 & & 0.11 \\
\hline Adenocarcinoma & 22.2 & & 35.7 & \\
\hline Neuroendocrine carcinoma & 50.0 & & 100.0 & \\
\hline Time to hepatic metastasis & & 0.37 & & 0.63 \\
\hline Synchronous & 22.2 & & 55.6 & \\
\hline Metachronous & 50.0 & & 66.7 & \\
\hline Primary surgery & & 0.87 & & 0.43 \\
\hline Yes & 25.0 & & 37.5 & \\
\hline No & 37.5 & & 80.0 & \\
\hline Other site metastasis & & 0.1 & & 0.91 \\
\hline Yes & 16.7 & & 50.0 & \\
\hline No & 100.0 & & 57.1 & \\
\hline Cycles of chemotherapy & & 0.28 & & 0.47 \\
\hline$<23$ & 50.0 & & 66.7 & \\
\hline$\geq 23$ & 22.2 & & 41.7 & \\
\hline Child-Pugh score & & 0.41 & & 0.26 \\
\hline 5 & 40.0 & & 66.7 & \\
\hline$\geq 6$ & 0.0 & & 50.0 & \\
\hline Hemoglobin (g/dL) & & 0.90 & & 0.47 \\
\hline$<11$ & 26.7 & & 53.3 & \\
\hline$\geq 11$ & 33.3 & & 75.0 & \\
\hline CEA (ng/mL) & & 0.90 & & 0.12 \\
\hline$<50$ & 33.3 & & 100.0 & \\
\hline$\geq 50$ & 26.7 & & 44.4 & \\
\hline Whole liver volume (mL) & & 0.94 & & 0.02 \\
\hline$<3,000$ & 66.7 & & 100.0 & \\
\hline$\geq 3,000$ & 20.0 & & 0.0 & \\
\hline Elevation of Child-Pugh score & & 0.04 & & 0.52 \\
\hline No & 40.0 & & 62.5 & \\
\hline Yes & 0.0 & & NR & \\
\hline Objective response & & 0.04 & & 0.43 \\
\hline Partial response or stable disease & 41.7 & & 53.6 & \\
\hline Progressive disease & 0.0 & & NR & \\
\hline
\end{tabular}

LPFS, local progression-free survival; PPFS, pain progression-free survival; ECOG, Eastern Cooperative Oncology Group; CEA, carcinoembryonic antigen; NR, not reached.

p-values in bold indicate statistical significance. 
A

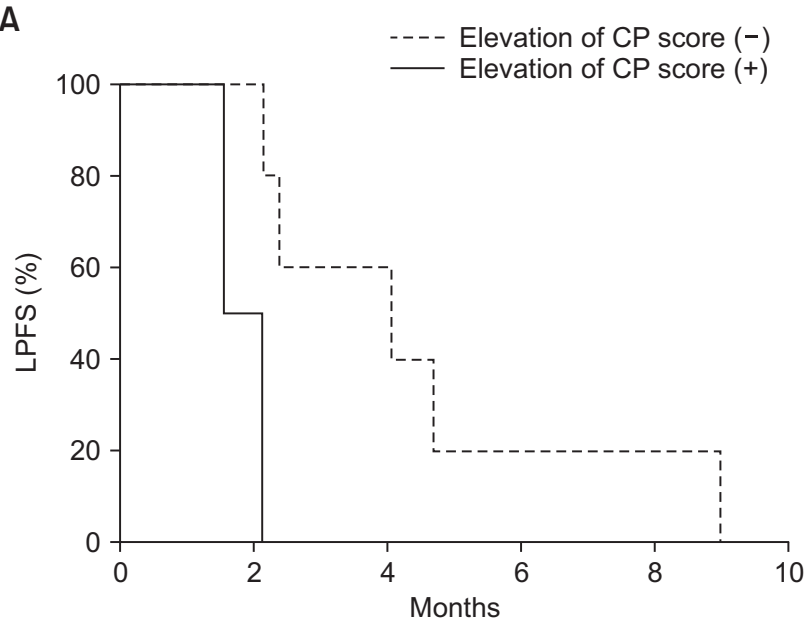

B

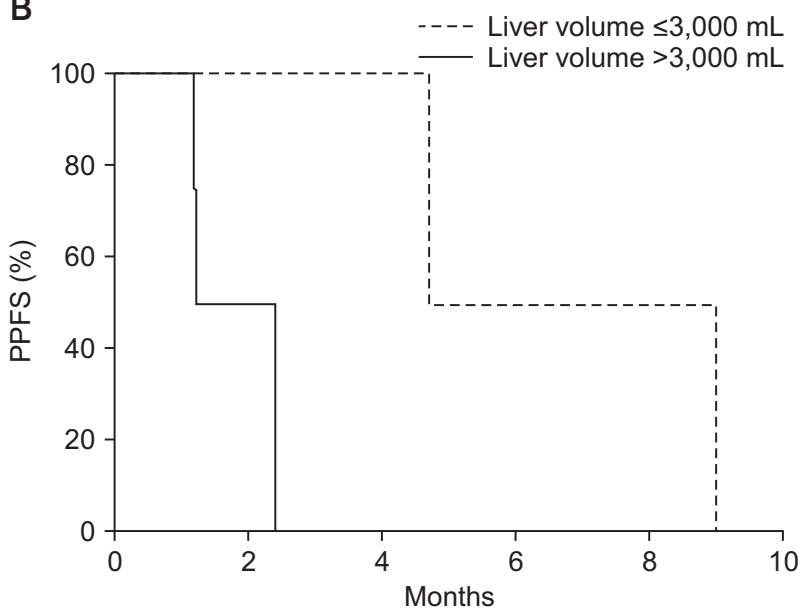

Fig. 5. Kaplan-Meier survival curves according to significant prognostic factors. (A) Elevation of Child-Pugh (CP) score at 1 month on local progression-free survival (LPFS) and (B) greater than 3,000-mL whole liver volume representing metastatic tumor volume on pain progression-free survival (PPFS).

tolerated combination treatments of low-dose WLI and hyperthermia. Grade III or higher adverse events developed because of either distant or intrahepatic disease progression. Although nearly half of the patients were not followed-up as scheduled, we were not aware of any radiation-induced hepatic toxicity.

Partial objective response was achieved in three patients after combined treatment. Considering that most of the enrolled patients received more than 10 cycles of chemotherapy, which may be a negative predictive factor of radiation response [21], the response rate was not disappointing. However, LPFS was still unacceptable at a median of 2.2 months. Therefore, additional systemic treatment in patients who show favorable response after treatment and recovered liver function could be an option. LPFS was slightly lower in patients with combined metastasis to sites other than the liver, so earlier application of WLI and hyperthermia could be another option.

Although HROOL was assessed in less than half of patients 2-3 months after treatment, HRQoL was maintained in assessed patients. Similar to $\mathrm{HRQOL}$, pain progression according to IBMCG criteria was delayed for up to a median of 4.7 months. In addition, symptom progression was clearly related to whole liver volume representing metastatic tumor burden. No pain progression was observed in patients who had a less than 3,000-mL whole liver volume during follow-up.

Therefore, earlier application of WLI and hyperthermia could be a valid option to obtain maximum local control without pain progression for patients with chemorefractory hepatic metastasis with smaller metastatic burden and metastasis confined only. But it should be considered that this study is not conclusively determine the real efficacy of combined WLI and hyperthermia because of the small sample size, single arm design, and early closure of the study.

This study had some limitations. Primarily, it ended early because of poor patient accrual and there was a high rate of follow-up loss. Only 40\% of patients were followed as planned due primarily to patient refusal. This phenomenon might be the result of eligibility criteria for the present study, which included patients who had not planned further treatment or wanted supportive care only. Therefore, our results are not sufficient to evaluate true efficacy and safety because of the small population and limited amount of evaluation data. Additionally, hyperthermia might be delivered suboptimally in patients with a larger tumor volume due to the limitation of an applicator. Most of all, analysis of prognostic factors might be limited due to the small sample size. Second, we could not assess the true temperature of the tumor area because of invasiveness and bleeding tendency in the enrolled patients.

In conclusion, combined WLI and hyperthermia in patients with numerous chemorefractory hepatic metastases from colorectal cancer might be promising without severe treatment-related toxicity, though we did not conclusively determine the real efficacy of combined WLI and hyperthermia over WLI alone in the present study because of the single arm design. However, early closure of this study and loss of most patients to follow-up limited our ability to evaluate true treatment effectiveness. Well-designed larger-scale prospective studies are warranted to evaluate the effectiveness and 
toxicity of WLI and hyperthermia for patients with numerous chemorefractory hepatic metastases from colorectal cancer.

\section{Conflict of Interest}

The hyperthermia system was rented from the Celsius42+ $\mathrm{GmbH}$ Company for prospective clinical studies using hyperthermia in combination with radiotherapy for primary and metastatic liver tumors. Grants for the clinical studies were provided by BiomediSyn, Inc.

\section{Acknowledgments}

This research was supported in part by a Samsung Medical Center grant (GF01130081) and by BiomediSyn Inc.

\section{Supplementary Materials}

Supplementary materials can be found via http://dx.doi. org/10.3857/roj.2016.34.1.34. Table 1. Energy escalation scheme of hyperthermia in WHITHER study. Table 2. The International Bone Metastases Consensus Group criteria. Table 3. Detailed chemotherapy regimens of the enrolled patients. Fig. 1. Kaplan-Meier survival curve of local progressionfree survival (LPFS) according to the presence of combined extrahepatic metastasis. Fig. 2. Kaplan-Meier survival curve of pain progression-free survival (PPFS) according to the pathology of primary tumor. Fig. 3. Kaplan-Meier survival curve of pain progression-free survival (PPFS) according to the initial level of the carcinoembryonic antigen (CEA).

\section{References}

1. Seo SI, Lim SB, Yoon YS, et al. Comparison of recurrence patterns between $\leq 5$ years and $>5$ years after curative operations in colorectal cancer patients. J Surg Oncol 2013; 108:9-13.

2. Bengmark S, Hafstrom L. The natural history of primary and secondary malignant tumors of the liver: I. the prognosis for patients with hepatic metastases from colonic and rectal carcinoma by laparotomy. Cancer 1969;23:198-202.

3. Geoghegan JG, Scheele J. Treatment of colorectal liver metastases. Br J Surg 1999;86:158-69.

4. Nordlinger $B$, Sorbye $H$, Glimelius $B$, et al. Perioperative chemotherapy with FOLFOX4 and surgery versus surgery alone for resectable liver metastases from colorectal cancer (EORTC Intergroup trial 40983): a randomised controlled trial. Lancet
2008;371:1007-16.

5. Hurwitz $H$, Fehrenbacher L, Novotny $W$, et al. Bevacizumab plus irinotecan, fluorouracil, and leucovorin for metastatic colorectal cancer. N Engl J Med 2004;350:2335-42.

6. Loupakis F, Cremolini C, Masi G, et al. Initial therapy with FOLFOXIRI and bevacizumab for metastatic colorectal cancer. N Engl J Med 2014;371:1609-18.

7. Michl M, Holtzem B, Koch J, et al. Metastatic colorectal cancer: analysis of treatment modalities and survival now and then Dtsch Med Wochenschr 2014;139:2068-72.

8. Cao R, Zhang S, Ma D, Hu L. A multi-center randomized phase Il clinical study of bevacizumab plus irinotecan, 5-fluorouracil and leucovorin (FOLFIRI) compared with FOLFIRI alone as second-line treatment for Chinese patients with metastatic colorectal cancer. Med Oncol 2015;32:325.

9. Cromheecke M, de Jong KP, Hoekstra HJ. Current treatment for colorectal cancer metastatic to the liver. Eur J Surg Oncol 1999;25:451-63.

10. Schefter TE, Kavanagh BD. Radiation therapy for liver metastases. Semin Radiat Oncol 2011;21:264-70.

11. Hoyer $M$, Swaminath $A$, Bydder $S$, et al. Radiotherapy for liver metastases: a review of evidence. Int J Radiat Oncol Biol Phys 2012;82:1047-57.

12. Yeo SG, Kim DY, Kim TH, Kim SY, Hong YS, Jung KH. Whole-liver radiotherapy for end-stage colorectal cancer patients with massive liver metastases and advanced hepatic dysfunction. Radiat Oncol 2010;5:97.

13. Leibel SA, Pajak TF, Massullo V, et al. A comparison of misonidazole sensitized radiation therapy to radiation therapy alone for the palliation of hepatic metastases: results of a Radiation Therapy Oncology Group randomized prospective trial. Int J Radiat Oncol Biol Phys 1987;13:1057-64.

14. Rao W, Deng ZS, Liu J. A review of hyperthermia combined with radiotherapy/chemotherapy on malignant tumors. Crit Rev Biomed Eng 2010;38:101-16.

15. Wust $P$, Hildebrandt $B$, Sreenivasa $G$, et al. Hyperthermia in combined treatment of cancer. Lancet Oncol 2002;3:487-97.

16. Borgelt BB, Gelber R, Brady LW, Griffin T, Hendrickson FR. The palliation of hepatic metastases: results of the Radiation Therapy Oncology Group pilot study. Int J Radiat Oncol Biol Phys 1981;7:587-91.

17. Ingold JA, Reed GB, Kaplan HS, Bagshaw MA. Radiation hepatitis. Am J Roentgenol Radium Ther Nucl Med 1965;93: 200-8.

18. Lawrence TS, Dworzanin LM, Walker-Andrews SC, et al. Treatment of cancers involving the liver and porta hepatis with external beam irradiation and intraarterial hepatic fluorodeoxyuridine. Int J Radiat Oncol Biol Phys 1991;20:55561.

19. Schattenberg JM, Schuchmann M, Galle PR. Cell death and 
hepatocarcinogenesis: dysregulation of apoptosis signaling pathways. J Gastroenterol Hepatol 2011;26 Suppl 1:213-9.

20. Park JS, Park HC, Choi DH, et al. Prognostic and predictive value of liver volume in colorectal cancer patients with unresectable liver metastases. Radiat Oncol J 2014;32:77-83.
21. Song DY, Jones RJ, Welsh JS, et al. Phase I study of escalating doses of low-dose-rate, locoregional irradiation preceding Cytoxan-TBI for patients with chemotherapy-resistant nonHodgkin's or Hodgkin's Iymphoma. Int J Radiat Oncol Biol Phys 2003;57:166-71. 